\title{
sciendo
}

Transport and Telecommunication, 2021, volume 22, no. 1, 87-97

Transport and Telecommunication Institute, Lomonosova 1, Riga, LV-1019, Latvia

DOI 10.2478/ttj-2021-0007

\section{A VISION-BASED REAL-TIME ADAPTIVE TRAFFIC LIGHT CONTROL SYSTEM USING VEHICULAR DENSITY VALUE AND STATISTICAL BLOCK MATCHING APPROACH}

\author{
Janak D. Trivedi ${ }^{1}$, Mandalapu Sarada Devi', Dhara H. Dave ${ }^{3}$ \\ ${ }^{1}$ Government Engineering College Bhavnagar, Electronics \& Communication Department, \\ Gujarat Technological University, \\ Gujarat, India \\ Trivedi_janak2611@yahoo.com
}

\begin{abstract}
In India, traffic control management is a difficult task due to an increment in the number of vehicles for the same infrastructure and systems. In the smart-city project, the Adaptive Traffic Light Control System (ATLCS) is one of the major research concerns for an Intelligent Transportation System (ITS) development to reduce traffic congestion and accidents, create a healthy environment, etc. Here, we have proposed a Vehicular Density Value (VDV) based adaptive traffic light control system method for 4-way intersection points using a selection of rotation, area of interest, and Statistical Block Matching Approach (SBMA). Graphical User Interface (GUI) and Hardware-based results are shown in the result section. We have compared, the normal traffic light control system with the proposed adaptive traffic light control system in the results section. The same results are verified using a hardware (raspberry-pi) device with different sizes, colors, and shapes of vehicles using the same method.
\end{abstract}

Keywords: Adaptive Traffic Light Control System (ATLCS), vehicular-density value (VDV), area of interest (AOI), raspberry-pi, Intelligent Transportation System (ITS), Graphical User Interface (GUI)

\section{Introduction}

The violation of traffic light control systems can cause an accident. According to the Ministry of Road Transport and Highways (MORTH), India ranked 1st among the number of road accident deaths across the 199 countries in the World Road Statistics in 2018. A total of 4,67,044 road accidents have been reported which is increased by $0.46 \%$ compared to the year 2017 . ATLCS does not reduce traffic accident but it's also avoided traffic collisions/congestions problem, reduce time-delay, etc. ATLCS requires continuous data or information from respective intersection points to the control unit at respective urban areas. Information Communication Technology (ICT) is useful for the development of smart cities and ITS.

In recent years, different image-video processing-based methodology is very useful for the development of an ITS. An autonomous vehicle requires this must technology for better reliability, safety, and flexibility. A Global Positioning System (GPS) opens the door for Vehicle to Vehicle (V2V), Vehicle to Infrastructure (V2I), Vehicle and handheld device (V2D), and Connected Vehicle Signal Control Strategy (CVSC). In this paper, we have used the image-video processing-based methodology for the development of ATLCS.

\subsection{Motivation}

Since the intelligent method of traffic light control system developed as per offline tool, TRANSYT (Robertson, 1969), Fixed time-plans for traffic junction, need to be enhanced and technology adaptive. Adaptive Traffic Light Control System is applied for real-time traffic information to minimize the delay of traffic, minimize the delay of pedestrians, minimize the delay of public transport, maximize reliability, minimize environmental impact, etc. A fixed time interval-based traffic light control system cannot accommodate day to day traffic congestion.

One of the limitations of Sensor-based optimizer to adequate with real-time traffic condition also the same time requires synchronization of individual Inductive Loop Detector (ILD) and requires additional installation if not installed earlier. In the case of a fixed-time traffic signal, we have to wait for a complete cycle of rotation with fixed time whether traffic is present or absent., whereas in case Adaptive Traffic Light Control System traffic time interval can be changed based on a different strategy or a traffic model. 
Different road-traffic models are discussed by Mfenjou et al. (2018). Maxband (Maximize Bandwidth) includes a two-way main road, with specifying passage time between the intersection point. Transyst (Traffic Network Study Tool) works on off-line optimization, which requires a lot of input parameters at intersection points. Prodyn is an adaptive traffic system for minimizing delay at intersection points. OPAC (Optimized Policies for Adaptive Control) works on a dynamic algorithm for minimizing stops at intersection points, the upgraded version of the system is OPAC-1,2,3 and 4. SCOOT (Split Cycle and Offset Optimization Technique) is an optimized control system that includes the duration of green light, offset between neighbouring intersections, and light cycles. This system also collects information about the number of vehicles per time interval. SCATS (Sydney Coordinated Adaptive Traffic System) is used information from sensors installed on the road for changing real-time traffic light plans at intersection points. Rhodes (Real-time Hierarchical Optimized Distributed Effective System) works on the concept of vehicular network. Insync has used image processing techniques for detecting vehicles and change traffic signals accordingly in real-time as per traffic demand.

\subsection{Organisation of the paper}

The remaining part of the paper is organized as follows: In Section 2, we have presented the Traffic Control System (TCS) using a different methodology. This part presents a survey of different image-video processing techniques, trends, and applications for the ITS. This part also indicates future scope from each of the respective articles. In section 3, we have presented the proposed method with a flowchart. In this part statistical block matching (SBMA) approach with pseudocode is explained. Section 4 and section 5 are indicating the GUI-based adaptive traffic control system and the Raspberry-pi (Hardware) -based adaptive traffic control system and results respectively. As for section 6, we have discussed the methodology and results with its objective outcomes. Finally, section 7 shows the conclusion and future scope of the presented work.

\section{Traffic Control Management System using a different methodology}

An image-video analysis is useful in traffic control management in terms of real-time an automatic parking management system, adaptive traffic control system, vehicle classification, automatic vehicle guidance system in a driverless vehicle, detection of static or movable objects, speed measurements, vehicle counting, incident detection such as an accident or congestion, automatic lane finding, different sign detection and recognition, and many more could be added in the list.

Kastrinaki et al. (2003) have explained a survey on a different aspect of video-processing techniques. Automatic Lane Finding (ALF) and Object detection for the stationary and moving camera are discussed with different approaches. ALF is discussed using (1) lane-region detection (2) feature and (3) model-driven approaches. Object detection is explained using (1) Thresholding (2) Multigrid identification of Region of Interest(ROI) (3) Edge-based detection (spatial differentiation) (4) Space signature (5) Background frame differencing (6) inter-frame differencing (7) Time signature (8) Feature aggregation and object tracking (9) Optical flow field (10) Motion parallax (11) Stereo vision (12) Inverse perspective mapping (13) 3D Modelling and forward mapping. In smart traffic management or adaptive traffic management is required (A) quickly changes and adapt system accordingly environmental changes and (B) automatic updating data or information from different sources.

Lozano et al. (2009) have presented Total Recognition by Adaptive Classification Experiments (TRACE) is described in this paper using feature selection/feature extraction with the help of the k-means clustering algorithm. The future scope from this article is finding real-time proper vehicle classification and vehicle counting. Video processing is a useful tool for finding vehicle velocity, stopped vehicles, travel time and queue length, traffic density for measurement of an automatic traffic control system, license plate detection, incident detection, vehicle size and vehicle shape for vehicle classification \& vehicle counting approaches, lane departure warning (or ALF), vehicle direction and traffic sign detection and recognition for driverless vehicle approach using Hough Transform in Bubenikova et al. (2012) for Advance Driver Assistance System (ADAS).

Mu et al. (2015) has explained the traffic light detection and recognition process, firstly from RGB (Red-Green-Blue) to HSV (Hue-Saturation-Value) conversion then apply the filtering process and finally for recognition, a histogram of oriented gradients (HOG) features and Support Vector Machine (SVM) is used. ADAS is required for the detection and recognition of not only circular traffic lights but also traffic lights in a horizontal and vertical arrangement. Trained Red, Yellow, and Green traffic light and 
Gaussian-like traffic light colour distribution are assumed. The limitation of the proposed method is that the method performs well only in daytime and false measurement increase during night time. Future work is the detection and recognition of more types of traffic lights.

Intelligent Transportation System (ITS) in a smart city or a smart road requires continuous monitoring of traffic data with good quality of image/video for the betterment of traffic engineering is explained by Bommes et al. (2016). The author analysed traffic with (1) Automatic Incident Detection (AID) of a dangerous traffic situation (2) Traffic count: direction detection of vehicles by Automatic Number/License Plate Recognition (ANPR/ALPR) (3) State recognition: classify traffic like congestion, slow traffic, and dense traffic. In that process Optical flow method and frame differencing method was used (4) Traffic management: video detection system for a dangerous object, shortening decision travel time, parking lot monitoring and management system, vehicle counting using Principal Component Analysis (PCA) (5) Measuring speed, toll collection, etc. Different application-wise technology matrix is mapped in terms of vision details as (a) Traffic webcam resolution (b) Traffic surveillance camera resolution (c) High-detail camera resolution. Future applications categorized as (i) Short-term, in which automatic recognition and automatic incident detection with the adaptive traffic control system is targeted (ii) Mid-term, in which Video-based technology for a better environment is focused and (iii) long-term, in which Communication with automated vehicles is set.

Chandan et al. (2017) has described as Connected Vehicle Signal Control (CVSC) for speed and location information using Global Positioning System (GPS) enabled vehicles. This method is compared with an adaptive traffic signal control simulation developed by PTV EPICS. Different traffic situations were tested in VISSIM 8 microscopic simulation tool. Limitation of Connected Vehicles (CV) during communication failure, which decreases the penetration rate. Future work is in the direction of real-time implementing the proposed method for adaptive traffic control management.

Makino et al. (2018) have given detail of Electric Toll Correction (ETC), Vehicle Information Communication Service (VICS), ETC 2.0 technology development as a part of the Intelligent Transportation System (ITS) in Japan. The combination of automatic driving system, ETC 2.0 is helpful for driver information about changing routes, changing lanes, avoid a collision, etc.

Traffic management project in Phnom Penh, the capital city of the Kingdom of Cambodia is discussed by S. Matsuoka (2018). Mainly two objectives are explained: (1) improve road traffic conditions with the help of a traffic control system, pavement markings, traffic signs, median dividers (2) transfer traffic management technology to local counterparts so that the project continues. First, this project is analysed before the implementation of the project: (i) the total number of registered vehicles (ii) traffic condition with a variety of vehicles, roads, driving behaviour, shortage of parking spaces (iii) pavement markings, traffic signs and other facilities. This project method is assessed with the listed benefits in the city like an increase in average speed and reduction in fuel consumption, emission, stop, delay, crash, travel time. The adaptive traffic control system with the same traffic volume for 2 intersection points with enabling and disabling during 1-hour time duration is measured and results are more prominent for enable condition of the adaptive traffic control system. Future challenges are listed as (a) proper operation and maintenance of the system (b) safety education and campaigning traffic rules among road users (c) expansion and up-gradation of the system.

Krzysztof et al. (2018) have explained smaller size cells compared to the Negel-Schreckenberg traffic model using cellular automaton (CA). The model is demonstrated with different driver behaviours: (1) The carefree driver (2) The driver with little skills (3) The standard driver. In this article, the countdown timer effect is useful for different driver approaches positively. Future work with more reliability of work, from a traffic light, mounted at intersection points.

Zhang et al. (2018) have described as a traffic decision using Case-Based Reasoning (CBR) and was verified by example. Different modules are considered for traffic management easy of operation, listed as (1) traffic information acquisition module (2) emergency scene information module (3) humancomputer interface module (4) emergency modelling module (5) aid decision-making module and (6) database module. Generally, the CBR case solving process includes four steps (a) Retrieve -the case (b) Reuse- case information and knowledge (c) Revise- if the large difference between previous and targeted case (d) Retain - the new solution. In this paper, case base characteristic attributes are mainly traffic peak, traffic type, place, whether, area, number of causalities, and number of damaged cars, and many more. Find similarities of various attributes in terms of (i) local similarity - enumerated, numerical, fuzzy (ii) overall similarity.

Rabbouch et al. (2018) has proposed an online video processing based automatic traffic control system model using Motion Intensity Index (MII) with a cumulating vision score frame by frame. After simple regression (linear regression) and wavelet regression (independent regression) forecast and convert MII into several vehicles for expected traffic flow counting and monitoring. Mfenjou et al. (2018) have explained different methodology-technology, application, and trends for developing an Intelligent 
Transportation System (ITS) in sub-Saharan Africa countries using Information Communication Technology (ICT). A hybrid system, a combination of four modules is discussed: (1) autonomous management of control system- to maximize communication coverage and a minimum number of control points (2) detection and diffusion of disturbance- to reduce congestion (3) dynamic route planning which is collaborated with disturbance detection (4) monitoring of sections- to reduce accidents in the road.

\section{The Proposed method with flow-chart}

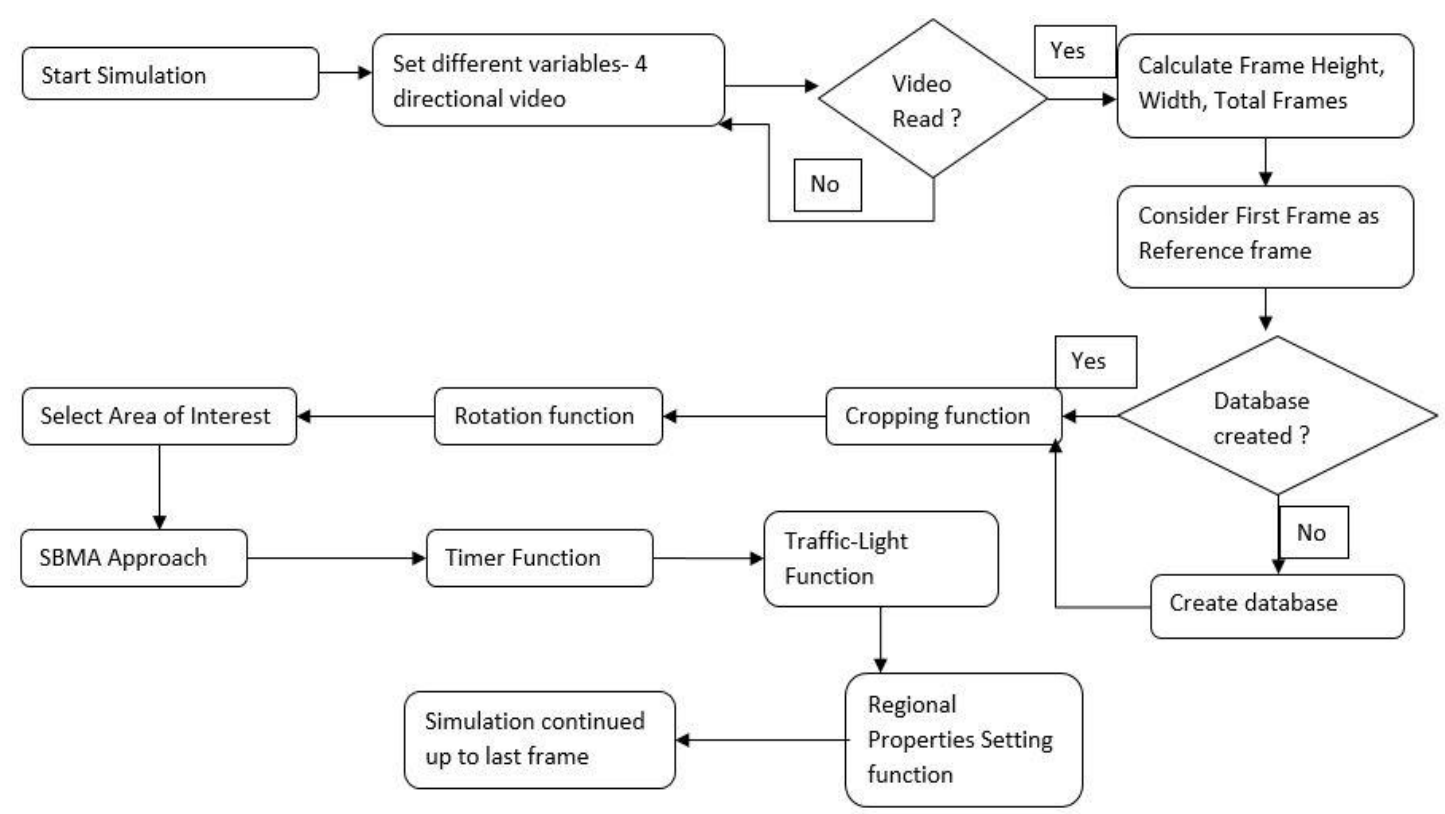

Figure 1. Flow-chart of the proposed method

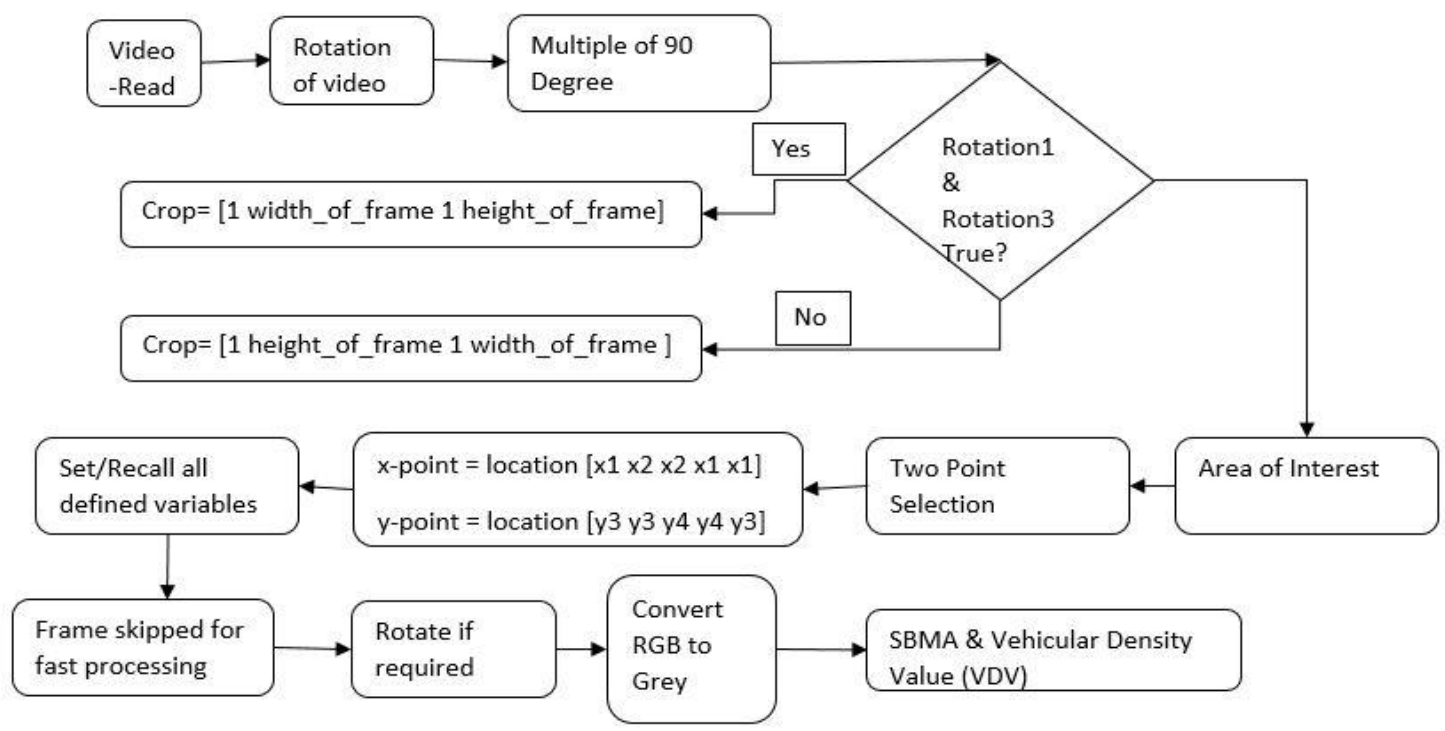

Figure 2. Flow-chart of rotation, area of interest, SBMA, VDV

A real-time ATLCS is developed using database management, selection of area of interest, selection of different image-video related properties for four-way intersection points, and combination of VDV and SBMA. Figure 1 represents the flowchart of the proposed method and figure 2 represents cropping, rotation, area of interest, SBMA, and VDV. In a normal traffic light control systems timing rotation and interval both are fixed irrelevant of any traffic condition, whereas in ATLCS visual information and vehicle density-based traffic timers are changing continuously. 
We have implemented our proposed method in software and hardware. GUI-based and Hardwar (Raspberry-pi)-based results are shown in result sections. SBMA, VDV method, and Pseudocode are represented in sections 3.1 and 3.2. Real-time video or location are inserted in GUI formation for video$1,2,3$ and 4 respectively. Rotation is one of the important selections for a proper selection of are of interest as well as for a vehicle density information. A simple $0^{\circ}, 90^{\circ}$ - left, $90^{\circ}$ - right, and $180^{\circ}$ rotation are required as per the selection of visual information. One of the novels approaches in the proposed method is the selection of a reference frame implemented using VDV. In the case of background subtraction method normally reference frame is selected, when there is no object in the image, whereas in the proposed method, liberty of selection for the reference frame. That first frame may contain a large number of objects, a small number of the object or none of them. We can also select an area of interest at any instant of time for real-time ATLCS measurements along with intersection points after cropping, rotation, and VDV is explained in section 3.1 with sets of equations and with pseudocode in section 3.2. This method works with spatial domain in the field of image-processing techniques.

\subsection{SBMA \& VDV}

$$
\begin{aligned}
& X_{\min }=\operatorname{Min}\left(x_{u l}, x_{l l}\right) \text { and } X_{\max }=\operatorname{Max}\left(x_{u r}, x_{l r}\right), \\
& Y_{\min }=\operatorname{Min}\left(\mathrm{y}_{u l}, \mathrm{y}_{l l}\right) \text { and } Y_{\max }=\operatorname{Max}\left(\mathrm{y}_{u r}, \mathrm{y}_{l r}\right), \\
& X_{d i s t}=X_{\max }-X_{\min }+1 \text { and } Y_{d i s t}=Y_{\max }-Y_{\min }+1, \\
& \text { If } \mathrm{y}_{u l}=\mathrm{y}_{u r} \longrightarrow x_{u l}=X_{d i s t} * \mathrm{y}_{u l} \text { else } x_{u l}=\mathrm{y}_{u l}: \frac{\mathrm{y}_{u r}-\mathrm{y}_{u l}}{X_{d i s t}-1} \vdots \mathrm{y}_{u r}, \\
& \text { If } \mathrm{y}_{l l}=\mathrm{y}_{l r} \longrightarrow x_{l l}=X_{d i s t} * \mathrm{y}_{l l} \text { else } x_{l l}=\mathrm{y}_{l l}: \frac{\mathrm{y}_{l r}-\mathrm{y}_{l l}}{X_{d i s t}-1} \vdots \mathrm{y}_{l r}, \\
& \text { If } x_{u l}=x_{u r} \longrightarrow y_{u l}=Y_{d i s t} * \mathrm{x}_{u l} \text { else } y_{u l}=x_{u l}: \frac{x_{u r}-x_{u l}}{Y_{d i s t}-1} \vdots x_{u r}, \\
& \text { If } x_{l l}=x_{l r} \longrightarrow y_{l l}=Y_{d i s t} * \mathrm{x}_{l l} \text { else } y_{l l}=x_{l l}: \frac{x_{l r}-x_{l l}}{Y_{d i s t}-1} \vdots x_{l r}, \\
& \theta=\sum_{j=1}^{4}(j-1) * 90, \\
& A=\sum_{i=1}^{n}\left[i_{r e f}-i_{c r o p}\right] \text { and mean }=\frac{1}{N} \sum_{i=1}^{N}\left[\mathrm{~A}_{i}\right], \\
& V D V=\operatorname{mean}(m e a n(A O I)), \\
& \text { if } \mathrm{R}_{i}=1 \rightarrow f_{c}=f_{c}+f_{s} \text { else } f_{c}=f_{c}+f_{l} .
\end{aligned}
$$

All the abbreviations are as mentioned below:

$X_{\text {min }}$ is minimum value of $X$-point,

$Y_{\text {min }}$ is minimum value of $Y$-point,

$x_{u l}$ is upper-left value of $x$-point,

$x_{l l}$ is lower-left value of $x$-point,

$x_{u r}$ is upper-right value of $x$-point,

$x_{l r}$ is lower-right value of $x$-point,

$X_{\text {dist }}$ is distance of $x$-point from a center location,

$R_{i}$ represents $i^{\text {th }}$ road location (here $i=1,2,3$ or 4 ),

$f_{c}$ represents current-Frame of the selected road,

$i_{r e f}$ represents reference-image intensity value,
$X_{\text {max }}$ is maximum value of $x$-point,

$Y_{\text {max }}$ is maximum value of $y$-point,

$y_{u l}$ is upper-left value of $y$-point,

$y_{l l}$ is lower-left value of $y$-point,

$y_{u r}$ is upper-right value of $y$-point,

$y_{l r}$ is lower-right value of $y$-point

$Y_{\text {dist }}$ is distance of $y$-point from a center location,

$f_{l}$ represents last-product calculation,

$f_{s}$ represents total number of skipped frames road,

$i_{\text {crop }}$ represents crop-image intensity value.

All the statistics block-matching approach and cropping measurements of the selected roads are done using a defined set of equations (1) to (7). After reading the video, rotate video with the desired 
direction using selected theta $(\theta)$ if required. Here, $\theta$ values are varied as $0^{\circ}, 90^{\circ}$-left, $90^{\circ}$-right, and $180^{\circ}$, which is derived using equation (8). Vehicle Density Value is measured using equation (9) and (10) with the combination of mean values of selected AOI row and column position. After selection of rotation, video, crop, area of interest, density values, GUI simulation provides ATLCS with timing and light control as well as remaining time of current junction and vehicle on-off time, to the next junction, i.e. If road-A indicates green signal then next 5 seconds is waiting time with yellow signal for the road- A and the same time with same yellow signal for Road-B provides information, 'about to start' this junction. In a fixed-time interval traffic light control system, the interval is fixed irrespective of vehicle information on the road, so there is not about-to start information needed. This way the proposed method also controls air pollution and gives better environmental conditions. In the process of GUI simulation when one road act as active at the same time another road should be passive. This is done using equation (11).

In this method, VDV is calculated using the mean of the selected AOI. First select, AOI for the selected roadside in the traffic signal. AOI calculates pixels' values information using matrix-vector operation for the selected region. The first time 'mean' operation applies to selected region which calculates mean of row and column pixel value. Then again 'mean' operation applies which provides single value. That single value represents density value information for the selected traffic roadside.

Nowadays, traffic surveillance systems available to monitor traffic in the city. In this article, $\theta$ represents the rotation of the camera situated at four-way intersection points in the city. $\theta$ value is varied as $0^{\circ}$ which represents traffic situation capture with camera position at traffic Road-A (fixed camera position), $\theta$ value is varied as $90^{\circ}$-left which represents traffic situation capture with camera position at traffic Road-B (camera rotates in $90^{\circ}$-left direction), and similar condition for the different choices of $\theta$. The single-camera situated at four-way intersection points with rotation facility can also monitor traffic signals at the traffic junction using the rotation $\theta$. The selection of $\theta$ is not a necessary step to implement this method.

\subsection{Pseudocode}

Time difference $=$ Time out for traffic light

If road $==1$ to 4 , Current threshold $=$ from final image 1 to 4

Otherwise, Current threshold $=0$

If $\mathrm{road}==1$ to 4 , Minimum threshold = Adaptive Threshold value

Otherwise, Minimum threshold $=0$

Traffic Light Condition

Waiting Time (Yellow color) $=5$ Second

Allow time $($ Green Light $)=15$ Second

Time difference $=$ Time out for traffic light

If road 1 to 4 selected but video not uploaded,

If, Time difference $<$ Required stop time

Set traffic time, with Stop=Red signal,

Otherwise, Time difference $>$ Required stop time

Start timer count

If road 1 then change road 2

If road 2 then change road 3

If road 3 then change road 4

If road 4 then change road 1

Color change

Current Threshold $>$ Adaptive Threshold value and Time difference $<$ Allow time

Traffic timer stops.

Current Threshold $<$ Adaptive Threshold value or Time difference $>$ Allow time

Traffic timer stops and goes to the next road.

Color Change Function in times box

Number $1=$ Red

Number2 $=$ Green

Number $3=$ Yellow

Display Traffic time

Four different roads

Use Number to string operation, display road wise respectively 


\section{Result: GUI-based adaptive traffic control system}

Figure 3 represents all roads A, B, C, D are blocked with a red signal. Figures 4 and 5 are shown with a yellow and a green light signal. For controlling air pollution, a wait signal is operated in two continuous roadsides. After completion of a green signal in the road-A side, a yellow signal indicates the reserved time for vehicles, at the same time the same reserved period indicates vehicle start time to Road-B. In the current scenario vehicles engine are mostly in on condition at the junction points, so this reserved period is helpful for vehicle engine ON/OFF time.

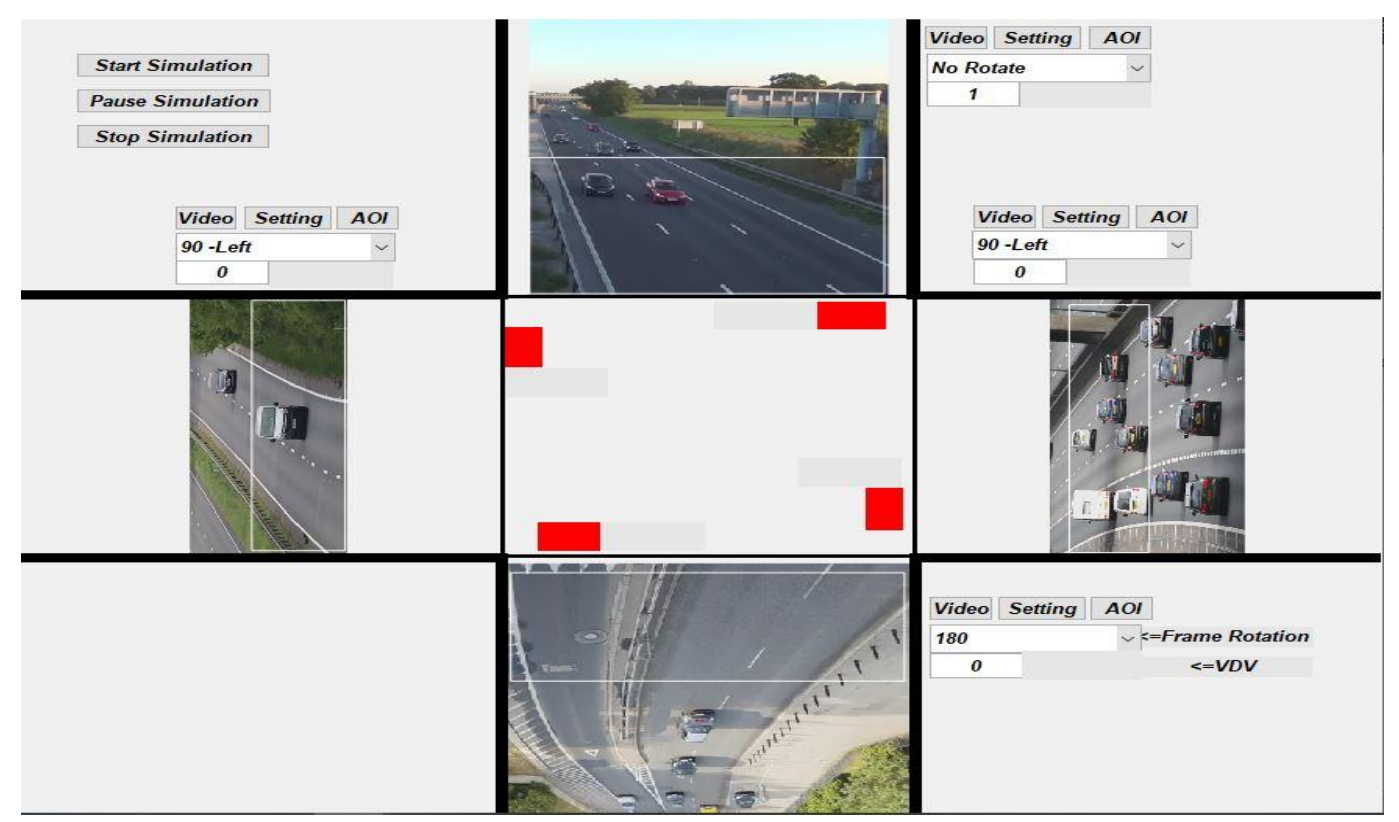

Figure 3. The starting phase with all red signal

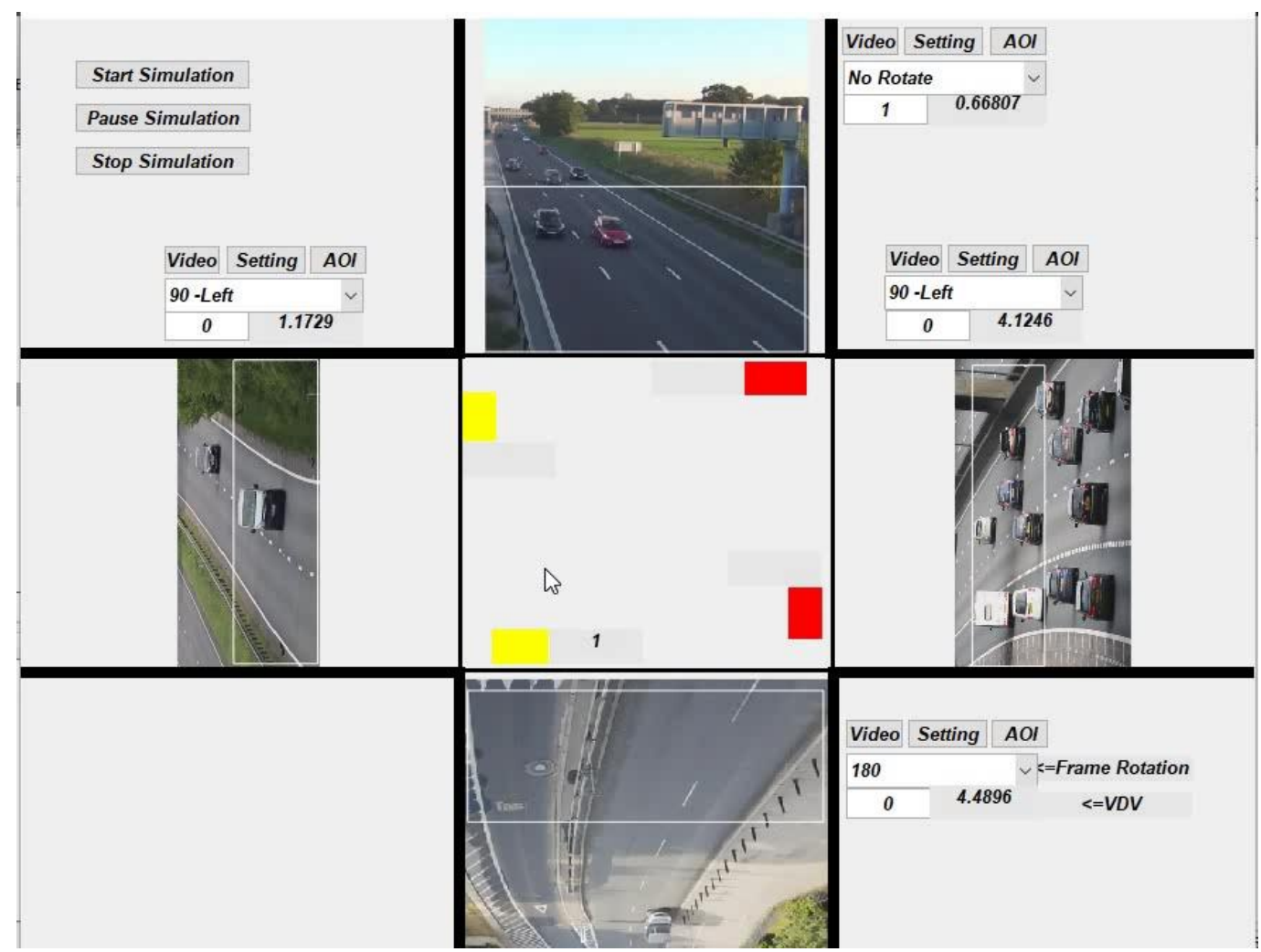

Figure 4. A yellow signal - waiting time 5 seconds 


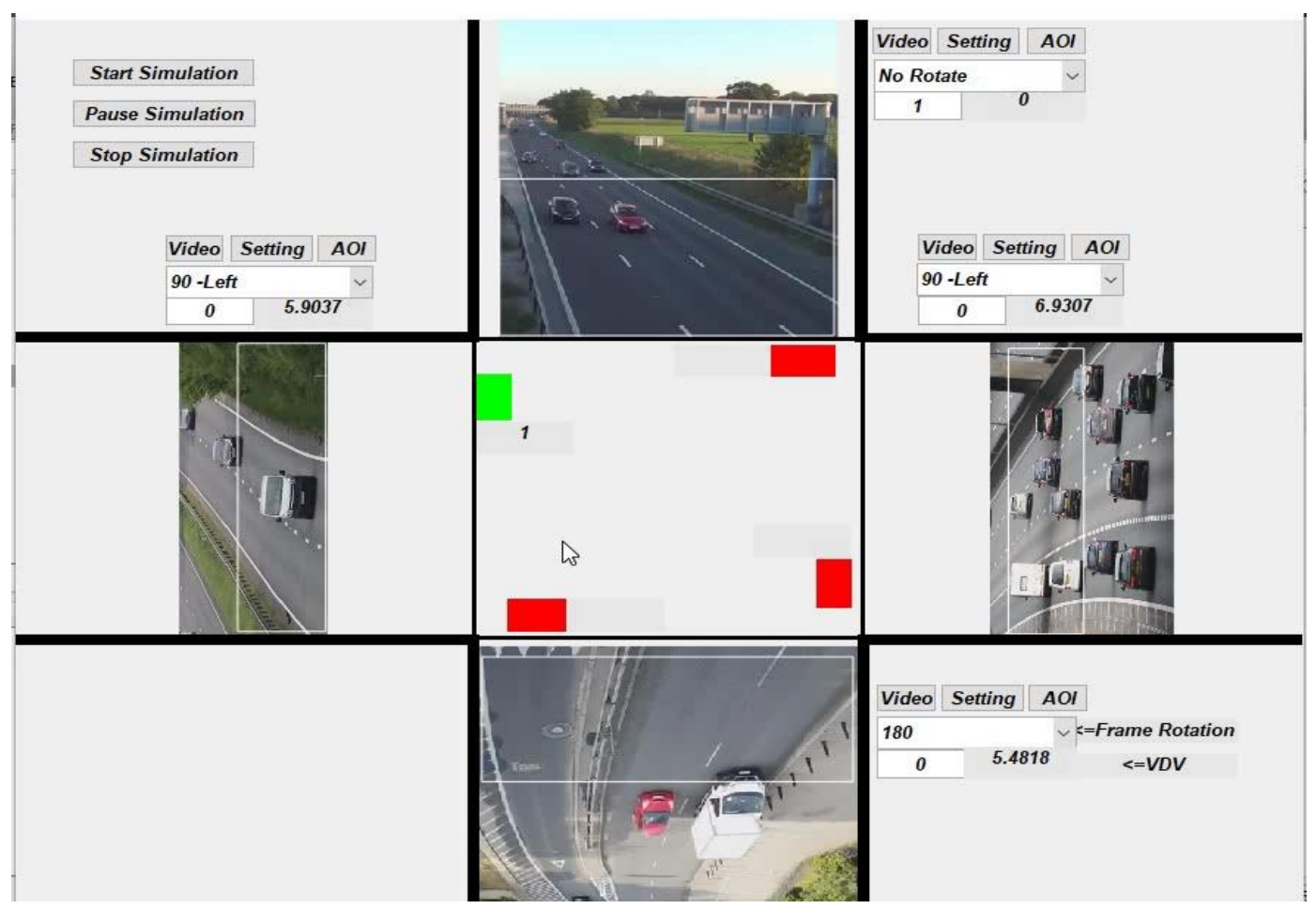

Figure 5. A green signal in one direction for 15 seconds

\section{Result: Raspberry-pi (Hardware)-based adaptive traffic control system module}

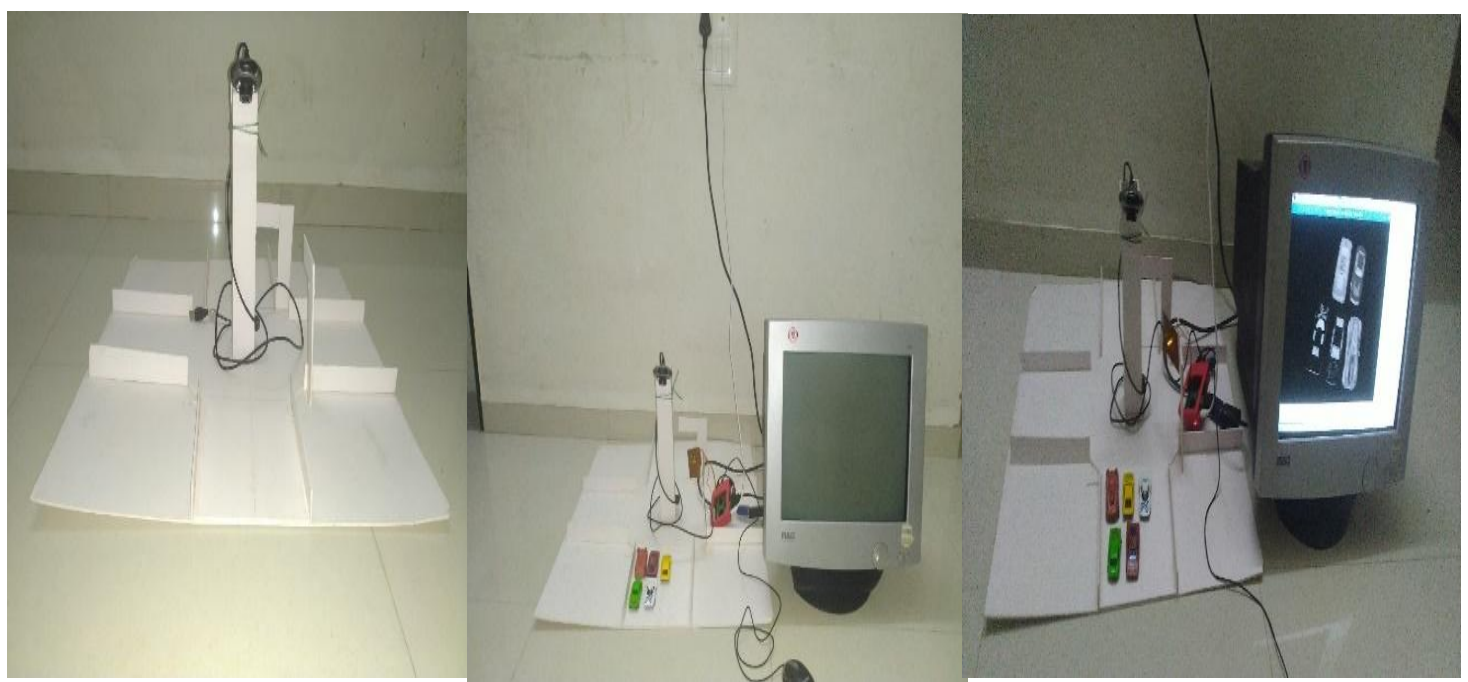

Figure 6. (a) Prototype model (b) Model connected using Raspberry-pi (c) Result for adaptive traffic control system using Raspberry-pi

The same method is uploaded to the raspberry-pi module. A hardware implementation of the raspberry-pi module is done using Simulink. During uploading the program to hardware first step is checking board parameter values with the connected environment. Then the next step is to select the build options, Serial Peripheral Interference (SPI) bus speed, external mode, and ports. With the help of deploying function, the same algorithm is programmed into raspberry-pi.

Figure 6 (a), (b), and (c) represent the prototype model and results for ATLCS. The proposed method is also applied in hardware with the use of the raspberry-pi 3B model. This hardware does not require any additional software installation; we can just plug into any monitoring system. Here, real-time ATLCS is managed in a prototype model with different vehicle sizes, shapes, colors, and numbers. 
3 different colors Light Emitting Diode (LED) with green, yellow, and red are used. Green LED for indication of 'Drive' status, yellow for 'Wait' status, and red for 'Stop'. Here, visual information is obtained using a USB camera. The display device is used for monitoring results. In monitoring screen waiting time, stop time, and drive time is continuously indicating with real-time changes in toy car replacement.

Table 1. Video Properties used in GUI simulation, resolution 640 X 360 frame rate in frames/second

\begin{tabular}{|l|l|l|l|l|l|l|}
\hline Road & $\begin{array}{l}\text { Total } \\
\text { Frame }\end{array}$ & $\begin{array}{l}\text { Frame } \\
\text { Rate }\end{array}$ & Rotation & Cropping & Area of Interest & $\begin{array}{l}\text { Density } \\
\text { value }\end{array}$ \\
\hline Road-A & 9000 & 30 & 180 & {$[270,635,10,355]$} & {$[1,360,10,145]$} & $\sim 5-10$ \\
\hline Road-B & 8992 & 29.97 & 90 -Left & {$[150,350,10,450]$} & {$[80,200,1,430]$} & $\sim 3-7$ \\
\hline Road-C & 7500 & 25 & 0 & {$[320,640,1,355]$} & {$[1,315,180,350]$} & $\sim 1-2$ \\
\hline Road-D & 7193 & 23.98 & 90 -Left & {$[1,360,1,640]$} & {$[35,185,15,640]$} & $\sim 18-25$ \\
\hline
\end{tabular}

${ }^{\$}$ Density values are selected from the probability of allowable time/vehicles for a particular road-side. It may be varied with different parameters.

VDV is depended mainly on AOI, vehicle color and size, number of vehicles. In the presented method, VDV is decided by trial and error method with maximum likelihood possibility for managing ATLCS. Different values of VDV can affect the number of passing vehicles, light, and timing of the TCS. The pseudocode represents the use of traffic light condition, timers, adaptive thresholding, color changes, and display traffic timer function for the proposed ATLCS.

Table 2. Compare the timing diagram for a different traffic control system

\begin{tabular}{|c|c|c|c|c|}
\hline \multirow[t]{2}{*}{ Traffic Control System } & \multicolumn{4}{|c|}{ Timing in second, stop time -40 , drive time -15 , wait time -5 . } \\
\hline & Road A & Road B & Road C & Road D \\
\hline \multirow{4}{*}{$\begin{array}{l}\text { Fixed Traffic Light Control } \\
\text { System }\end{array}$} & $15+5$ & 40 & 40 & 40 \\
\hline & 40 & $15+5$ & 40 & 40 \\
\hline & 40 & 40 & $15+5$ & 40 \\
\hline & 40 & 40 & 40 & $15+5$ \\
\hline \multirow{4}{*}{$\begin{array}{l}\text { Fixed Traffic Light Control } \\
\text { System (Without VDV, With } \\
\text { Remaining time, full traffic) }\end{array}$} & $15+5$ & $40-5$ & 40 & 40 \\
\hline & 40 & $15+5$ & $40-5$ & 40 \\
\hline & 40 & 40 & $15+5$ & $40-5$ \\
\hline & $40-5$ & 40 & 40 & $15+5$ \\
\hline \multirow{4}{*}{$\begin{array}{l}\text { Fixed Traffic Light Control } \\
\text { System (With VDV*, With } \\
\text { Remaining time, full traffic) }\end{array}$} & $15+5$ & $30-5$ & $45-5$ & $60-5$ \\
\hline & $60-5$ & $15+5$ & $30-5$ & $45-5$ \\
\hline & $45-5$ & $60-5$ & $15+5$ & $30-5$ \\
\hline & $30-5$ & $45-5$ & $60-5$ & $15+5$ \\
\hline \multirow{4}{*}{$\begin{array}{l}\text { Adaptive (With VDV*, With } \\
\text { Remaining time, controlled } \\
\text { traffic) }\end{array}$} & $10+5$ & $25-5$ & $40-5$ & $55-5$ \\
\hline & $57-5$ & $12+5$ & $27-5$ & $42-5$ \\
\hline & $38-5$ & $53-5$ & $8+5$ & $23-5$ \\
\hline & $29-5$ & $44-5$ & $59-5$ & $14+5$ \\
\hline
\end{tabular}

* As per changes in VDV values complete cycle rotation of road A-B-C-D-A also vary, it provides always a higher number of cycle rotation compares to normal traffic light control system.

\section{Discussion}

We have proposed a vision based constant ATLCS for a 4-way crossing point, utilizing a determination of turn, AOI, VDV, and SBMA. Figure (1) and figure (2) shows a flowchart of the proposed technique. Figure (3) to figure (6) speaks to Graphical User Interface (GUI) based simulation results for 4-way intersection point (Sub-figures are turned according to single-camera see with a fixed situation at crossing point). Similar outcomes are additionally checked to utilize equipment (raspberry-pi) with various sizes, colours, and shapes of vehicles utilizing a similar technique. A prototype module appears in figure 6(a), figure 6(b) shows associated set-up of equipment and screen, and in figure 6(c) shows the result for versatile traffic light control framework utilizing equipment (raspberry-pi) gadget with "Pause" (for 15 second or no vehicle out and about) and "Drive" (for 45 seconds or most extreme number of vehicles for particular side) message on the screen. S. Srivastava et al. (2016) expresses ATLTC with a period stream outline for a solitary side, though here the proposed technique for a 4-way intersection point. In the outcome segment we have compared, the typical traffic light control framework with the proposed versatile traffic light control framework in Table 2 with the assistance of the span of time-stamps. 
The proposed technique is expelled restriction of fixed time traffic light control framework structured by offline tool TRANSYT (1969). This technique likewise controls traffic at the 4-way crossing point focuses, which is an improved adaptation of the ATLTC (2016). Table 1 shows various 4video properties and Table 2 shows the correlation of the diverse traffic control frameworks with the planning cycle.

\section{Conclusions \& Future-scope}

We present, vision-based continuous ATLCS. This strategy gives an improvement as far as a more noteworthy number of complete cycle revolution for road, for example, A-B-C-D-A compared with the fixed traffic-light control framework. If there is no vehicle or few vehicles present at any single (roadside-A), opposite side streets (road-side B, C, D) get profited in the wake of discovering relative vehicle density data. As appeared in table 2, road A, B, C, and D decrease 5, 3, 7, and 1-second cycle revolution individually compared to fixed time traffic light control system. The proposed strategy limits traffic delay which helps to reduce traffic congestions, environmental effects. The reserved-time idea is likewise valuable for the information on vehicle on-off time, it might assist with lessening fuel utilization just as a superior natural condition. The constraint of this work is, the proposed technique relies upon the vision framework introduced at convergence focuses that have variable video properties.

In a future work try to installed the proposed method for any junction points to obtain the outcomes and effect on the same.

\section{References}

1. Robertson, D. I. (1969) TRANSYT: A Traffic Network Study Too, RRL Report LR 253, Road Research Laboratory Crowthorne, Berkshire.

2. Kastrinaki, V., Zervakis, M., Kalaitzakis, K. (2003) A survey of video processing techniques for traffic applications. Image Vis. Comput., 21(4), pp. 359-381.

3. Lozano, A., Manfredi, G., Nieddu, L. (2009) An algorithm for the recognition of levels of congestion in road traffic problems. Math. Comput. Simul., 79(6), pp. 1926-1934.

4. Bubeníková, E., Muzikáŕová, L., Halgaš, J. (2012) Application of image processing in intelligent transport systems. IFAC Proc. Vol., 11(1), pp. 53-56.

5. Mu, G., Xinyu, Z., Deyi, L., Tianlei, Z., Lifeng, A. (2015) Traffic light detection and recognition for autonomous vehicles. J. China Univ. Posts Telecommun., 22(1), pp. 50-56.

6. Nemade, B. (2016) Automatic Traffic Surveillance Using Video Tracking. Procedia Comput. Sci., 79, pp. 402-409.

7. Małecki, K. (2016) The Importance of Automatic Traffic Lights Time Algorithms to Reduce the Negative Impact of Transport on the Urban Environment. Transp. Res. Procedia, 16(March), pp. 329-342.

8. Bommes, M., Fazekas, A., Volkenhoff, T., Oeser, M. (2016) Video Based Intelligent Transportation Systems - State of the Art and Future Development. Transp. Res. Procedia, 14, pp. 4495-4504.

9. Srivastava, S. et al. (2016) Adaptive Traffic Light Timer Control (ATLTC), Adaptive Traffic Light Timer Control (ATLTC) - NERD.

10. Chandan, K., Seco, A. M., Silva, A. B. (2017) Real-time Traffic Signal Control for Isolated Intersection, using Car-following Logic under Connected Vehicle Environment. Transp. Res. Procedia, 25, pp. 1610-1625.

11. Kotov, A., Pospelov, P. (2017) Engineering Tools and Methods of Estimation of Traffic Capacity Using Mobile Video Monitoring. Transp. Res. Procedia, 20, pp. 347-354.

12. Makino, H., Tamada, K., Sakai, K., Kamijo, S. (2018) Solutions for urban traffic issues by ITS technologies. IATSS Res., 42(2), pp. 49-60.

13. Nagy, A. M., Simon, V. (2018) Survey on traffic prediction in smart cities, Pervasive Mob. Comput., 50, pp. 148-163.

14. Aibin, M. (2018) Traffic prediction based on machine learning for elastic optical networks. Opt. Switch. Netw., 30, pp. 33-39.

15. Rabbouch, H., Saâdaoui, F., Mraihi, R. (2018) A vision-based statistical methodology for automatically modeling continuous urban traffic flows. Adv. Eng. Informatics, 38, pp. 392-403.

16. Matsuoka, S. (2018) Traffic management project in Phnom Penh. IATSS Res., 42(4), pp. 180-189.

17. Mfenjou, M. L., Abba Ari, A. A., Abdou, W., Spies, F., Kolyang (2018) Methodology and trends for an intelligent transport system in developing countries, Sustain. Comput. Informatics Syst., 19, pp. 96-111. 
18. Zhang, H., Dai, G. L. (2018) Research on traffic decision making method based on image analysis case-based reasoning. Optik (Stuttg)., 158, pp. 908-914.

19. Małecki, K., Iwan, S. (2019) Modeling traffic flow on two-lane roads with traffic lights and countdown timer, Transp. Res. Procedia, 39, no. 2018, pp. 300-308.

20. Dodsworth, Joel Andrew (2018) The application of vehicle classification, vehicle-to-infrastructure communication and a car-following model to single intersection traffic signal control, Ph.D. thesis, University of Leeds. 